\title{
Subparacompactness in Cartesian products of generalized ordered topological spaces ${ }^{(1)}$
}

by

\section{K. Alster (Warszawa)}

Abstract. By a $\mathrm{GO}_{\delta}$-space we understand a space with a $G_{\delta}$-diagonal which can be embedded in a linearly ordered topological space. In this paper some results about subparacompactness in Cartesian products of $\mathrm{GO}_{\delta}$-spaces are proved. Among others it is shown that the product of two Lindelöf $\mathrm{GO}_{\delta}$-spaces is hereditarily subparacompact if and only if the Continuum Hypothesis holds. The Cartesian product of three $\mathrm{GO}_{\delta}$ if and only if the Continuum Hypothesis holds. The Cartesian product of this theorem is not true for products of four spaces. A necessary and sufficient condition for the product of four $\mathrm{GO}_{\delta}$-spaces to be subparacompact is that each pair of disjoint closed subsets of this product can be separated by $G_{5}$-sets.

0. Introduction. The aim of this paper is to give some results about subparacompactness $\left({ }^{2}\right)$ in Cartesian products of generalized ordered topological spaces (abbreviated GO-spaces) $\left(^{3}\right)$.

Let us notice that many important examples connected with paracompactness in Cartesian products were obtained in the class of generalized topological spaces (see [12], [9], [10] and [11]). These examples have an additional property, namely they have $G_{\delta}$-diagonals.

Our paper is organized in the following way: In section 1 we give basic definitions, section 2 contains technical lemmas and propositions which are used later, and the most important section 3 is devoted to a study of problem under what conditions the product $\boldsymbol{P} X_{\boldsymbol{s}}$ of GO-spaces with $G_{\delta}$-diagonals (abbreviated $\mathrm{GO}_{\delta}$-spaces) is subparacompact or hereditarily subparacompact.

Let us recall that in [1] an example of a generalized ordered topological space $X$ such that $X \times X$ is not subparacompact is constructed; if we assume the Continuum Hypothesis then the space $X$ can be defined in such a way that the weight of $X$ is $\kappa_{1}$. In both cases the space $X$ has not

(1) This is a part of the authors doctoral dissertation written under the supervision of Professor R. Engelking.

(2) Subparacompactness has been extensively studied by D. Burke (in [3])

(s) GO-spaces have been investigated by D. Lutzer (in [7]). 
a $G_{\delta}$-diagonal, so our restriction to generalized ordered topological spaces with $G_{\delta}$-diagonals is natural in this context.

We shall often use the following theorem proved by $\mathrm{D}$. Lutzer in [7] (Theorem 4.3):

(0.1) THEOREM. Every $\mathrm{GO}_{\delta}$-space is hereditarily paracompact.

We adopt the terminology and notation of [4].

\section{Basic definitions.}

(1.1) Defrntrion. A space $X$ is subparacompact (finitely subparacompact or countably subparacompact) if every (finite or countable) open covering has a $\sigma$-discrete closed refinement $\left(^{4}\right)$.

(1.2) Remark. Let us notice that $X$ is finitely (countably) subparacompact if and only if every finite (countable) open covering has a shrinking consisting of $F_{\sigma}$-sets.

(1.3) Remark. It is easy to see that finite subparacompactness is a generalization of normality, namely a space $X$ is finitely subparacompact if and only if for each pair $A, B$ of disjoint closed sets there exist disjoint $G_{8}$-sets $V$ and $U$ such that $A \subset V$ and $B \subset U$.

(1.4) Definition. By GO $(K, \leqslant)$ we denote the class of GO-spaces of the form $(K, \tau, \leqslant)$, where $(K, \leqslant)$ is a fixed linearly ordered sot (see [7], Definition 2.1).

(1.5) Definition. We say that $X$ is a $\mathrm{GO}_{8}$-space if $X$ is a $\mathrm{GO}$-space and has a $G_{\delta}$-diagonal.

(1.6) Definition. By $\mathrm{GO}_{\delta}(K, \leqslant)$ we mean the class of $\mathrm{GO}_{\delta}$-spaces of the form $(K, \tau, \leqslant)$, where $(K, \leqslant)$ is a fixed linearly ordered set.

(1.7) Definimion. By an interval in a generalized ordered space $(X, \tau, \leqslant)$ we mean any subset $U$ of the form:

$$
J=\{x \in X: a<x \leqslant b\}, \quad U=\{x \in X: a \leqslant x<b\}
$$

$$
\text { or } \quad U=\{x \in X: a<x<b\} \text {. }
$$

A one point subset of $X$ which is not an interval will be called a degenerate interval.

(1.8) Definition. We say that $\mathfrak{B}_{a}$ is a canonical base at a point $a \in X$, where $X$ is a GO-space, if $\mathfrak{B}_{a}$ is a base at the point a consisting of intervals or degenerate intervals and satisfying the following conditions:

(1) if $a$ is an isolated point then $\mathfrak{B}_{a}=\{\{a\}$,

(2) if $a$ is an accumulation point and $\{x \in X: x \geqslant a\}$. is an open subset of $X$ then $U \subset\{x \in X: x \geqslant a\}$ for every $U \in \mathfrak{B}_{a}$,

${ }^{(4)}$ We say that $\mathfrak{U}=\left\{U_{s}\right\}_{s \in S}$ is a refinement of $\mathfrak{B}=\left\{V_{t}\right\}_{t \in T}$ if for every $s \in S$ there exists a $t_{\mathfrak{t}} \in T$ such that $U_{\mathfrak{s}} \subset V_{t_{s}}$ and $\bigcup \mathfrak{H}=\bigcup \mathfrak{B}$.
(3) if $a$ is an accumulation point and $\{x \in X: x \leqslant a\}$ is an open subset of $X$ then $U \subset\{x \in X: x \leqslant a\}$ for every $U \in \mathfrak{B}_{a}$.

2. Technical lemmas. We start with a theorem which is an unpublished result of T. Przymusiński. The proof which we are going to present is simpler than the original one and is based on the Bing metrization theorem (see [2]): a regular space is metrizable if and only if it is collectionwise normal and developable $\left.{ }^{5}\right)$.

(2.1) THEOREM. If $X$ is a $\mathrm{GO}_{\delta}$-space from the class $\mathrm{GO}(K, \leqslant)$, where $K$ is a fixed linearly ordered set, then there exists a metrizable space $M$ which belongs to $\mathrm{GO}(K, \leqslant)$ such that the topology of $M$ is weaker than the topology of $X$.

Proof. Let $\left\{G_{n}\right\}_{n=1}^{\infty}$ be a sequence of open subsets of $X \times X$ such that $\bigcap_{n=1}^{\infty} G_{n}=\{(x, x): x \in X\}$. Let $U_{n x}$ be, for $x \in X$ and $n \in N$ (N denotes the set of natural numbers), an arbitrary open subset of $X$ which satisfies the following conditions:

(4) $U_{n x} \in \mathfrak{B}_{x}, \quad$ where $\mathfrak{B}_{x}$ is a eanonical base at the point $x$,

$$
\begin{gathered}
U_{n x} \times U_{n x} \subset G_{n}, \\
U_{n+1, x} \subset U_{n x} .
\end{gathered}
$$

From (5) it follows that

(7) $\bigcap_{n=1}^{\infty} \mathrm{St}\left(x, \mathfrak{U}_{n}\right)=\{x\}, \quad$ where $\quad \mathfrak{U}_{n}=\left\{U_{n x}\right\}_{x \in X} \quad$ for $n=1,2, \ldots$

Let us put for $x \in X$ and $n=1,2 \ldots$

$$
\begin{aligned}
& x_{n}^{-}=\left\{\begin{array}{l}
x \quad \text { if } \quad \text { inf } \operatorname{St}\left(x, \mathfrak{U}_{n}\right)=x, \\
\text { an arbitrary point of St }\left(x, \mathfrak{U}_{n}\right) \cap\{z \in X: z<x\} \\
\text { if this set is not empty . }
\end{array}\right. \\
& x_{n}^{+}=\left\{\begin{array}{l}
x \quad \text { if } \quad \sup \operatorname{St}\left(x, \mathfrak{U}_{n}\right)=x, \\
\text { an arbitrary point of } \operatorname{St}\left(x, \mathfrak{U}_{n}\right) \cap\{z \in X: z>x\} \\
\text { if this set is not empty } .
\end{array}\right.
\end{aligned}
$$

(inf $A$ and $\sup A$ for $A \subset K$ are defined with respect to the linear order of $K$ ).

As a base at any point $x \in M=K$ we take the family

$$
\left\{\left\{y \in M: x_{n}^{-}<y<x_{n}^{+}\right\} \cup\{x\}\right\}_{n=1}^{\infty} .
$$

(5) A space $X$ is developable if there exists a sequence $\left\{\mathfrak{U}_{n}\right\}_{n=1}^{\infty}$ of open coverings such that for every $x \in X$ and its open neighborhood $U$ there is a natural number $n$ satisfying $\mathrm{St}\left(x, \mathfrak{U}_{n}\right) \subset \bar{U}$. 
Let us put $\mathfrak{H}_{n}=\left\{\operatorname{Int}_{M} U\right\}_{U \in \mathfrak{X x}_{n}}$ where $\operatorname{Int}_{M} U$ denotes the interior of $U$ relatively to $M$.

From the Bing metrization theorem, Theorem 2.9 from [7] and the fact that a linearly topological space is hereditarily collectionwise normal (see [13]) it follows that the proof of Theorem (2.1) will be completed if we show that

(9) the topology of $M$ is weaker than the topology of $X$,

(10) $\left\{\mathfrak{H}_{n}\right\}$ is a development of $M$.

Ad (8). The base of $M$ consists of intervals and degenerate intervals. Let $x \in U$, where $U$ is an arbitrary set of the form $\{x \in K: a<x<b\}$, or $\{x \in K: a<x\}$, or else $\{x \in K: x<b\}$. Let $E=\{x \in K: x=\inf U$ if $\inf U$ exists or $x=\sup U$ if $\sup U$ exists $\}$. There exists $n(x) \in N$ such that $\operatorname{St}\left(x, U_{n(x)}\right) \subset K \backslash E$ (see (7) and (6)). It is easy to see that $\left\{x \in K: x_{n(x)}^{-}\right.$ $\left.<x<x_{n(x)}^{+}\right\} \subset U$.

Ad (9). Let us notice that if the set

$$
U=\{x \in K: x \leqslant a\} \quad(U=\{x \in K: x \geqslant a\})
$$

is open in $M$, then it is open in $X$ and because $M, X \in \mathrm{GO}(K, \leqslant)$, the topology of $M$ is weaker than the topology of $X$.

Ad (10). From (4) it follows that if $x \notin \bigcup\left\{\left(\operatorname{Int}_{K} U\right)\right\}_{U \in \mathfrak{U}_{n}}$ then $U_{n x}$ $=\operatorname{St}\left(x, \mathfrak{U}_{n}\right)$, therefore $x \in \operatorname{Int}_{M} U_{n x}$ and $\mathfrak{S}_{n}$ is the covering of $M$ for $n=1,2, \ldots$

The proof of (10) will be finished if for

$$
U=\left\{x^{\prime} \in M: x_{n}^{-}<x^{\prime}<x_{n}^{+}\right\} \cup\{x\} .
$$

we find a natural number $m(x)$ such that $\operatorname{St}\left(x, \mathfrak{U}_{m(x)}\right) \subset U$. Let us put

$$
\begin{aligned}
& m\left(x_{n}^{-}\right)=\left\{\begin{array}{l}
n \quad \text { if } \quad x_{n}^{-}=x, \\
\text { an arbitrary natural number } m \text { such that } \\
\operatorname{St}\left(x, \mathfrak{U}_{m}\right) \subset K \backslash\left\{x_{n}^{-}\right\} \quad \text { if } x_{n}^{-} \neq x .
\end{array}\right. \\
& m\left(x_{n}^{+}\right)=\left\{\begin{array}{l}
n \quad \text { if } x_{n}^{+}=x, \\
\text { an arbitrary natural number } m \text { such that } \\
\operatorname{St}\left(x, \mathfrak{U}_{m}\right) \subset K \backslash\left\{x_{n}^{+}\right\} \quad \text { if } x_{n}^{+} \neq x .
\end{array}\right.
\end{aligned}
$$

The number $m(x)=\max \left\{m\left(x_{n}^{+}\right), m\left(x_{n}^{-}\right)\right\}$has the required properties.

A metrizable $\mathrm{GO}_{\delta}$ - space $M$ which satisfies conditions of Theorem (2.1)

will be called a space associated with the $\mathrm{GO}_{\delta}$-space $X$.

The following lemmas will play a fundamental role in the sequel.

(2.2) LEMma. If $M=(K, \tau, \leqslant)$ is a metrizable GO-space and If a family of intervals, then $\cup \mathfrak{U}$ is an $\mathbb{F}_{\sigma}$-set in $M$.
Proof. Let us put

$$
V=\bigcup\left\{\operatorname{Int}_{M} U: U \in \mathfrak{U}\right\} \quad \text { and } \quad \mathfrak{U}_{n}=\{U \in \mathfrak{U}: \operatorname{diam} U>1 / n\},
$$

where diam is taken with respect to the fixed metric $\varrho$ of $M$, and $n=1,2, \ldots$ It is easy to see that $Z_{n}=\left(\cup \mathfrak{U}_{n}\right) \backslash V$ is closed in $M$, for $n=1,2, \ldots$, and that $V$ is an $F_{\sigma}$-set in $M$. The proof of Lemma (2.2) follows from the equality $U=\bigcup_{n=1}^{\infty} Z_{n} \cup V$.

(2.3) CoRollary. If $X$ is a $\mathrm{GO}_{\delta}$-space then $X^{d}$ (the derived set) is perfect, i.e. every open subset of $X^{d}$ is an $F_{\sigma^{-}}$set in $X^{d}$.

(2.4) Lemma. If $\mathfrak{U}$ is an open family in $X_{1} \times \ldots \times X_{n}$, where $X_{i}$ is a $\mathrm{GO}_{\delta}$-space for $i=1,2, \ldots, n$, such that every $U \in \mathfrak{U}$ is of the form $U=U_{1} \times \ldots \times U_{n}$, where one of the sets $U_{1}, \ldots, U_{n}$ is an interval in the corresponding $X_{i}$ and others are open in $M_{j}$ associated with $X_{j}$ for $j \neq i$ then $\mathfrak{U}$ has a $\sigma$-discrete refinement.

Proof. Let $\mathfrak{U}_{i}=\left\{U_{1} \times \ldots \times U_{n} \in \mathfrak{U}: U_{j}\right.$ is an open subset of $M_{j}$ for $j \neq i\}$. The proof will be finished if we define a $\sigma$-discrete refinement $\mathfrak{E}_{i}$ of $\mathfrak{U}_{i}$ for $i=1,2, \ldots, n$. We can assume without loss of generality that $i=1$. Let $\mathfrak{B}$ be a $\sigma$-discrete base of $M_{2} \times M_{3} \times \ldots \times M_{n}$ and $\mathfrak{B}$ an open refinement of $\mathfrak{U}_{1}$ such that if $V \in \mathfrak{B}$ then $V=V_{1} \times V_{2}$, where $V_{1}$ is an interval in $\bar{X}$ and $\nabla_{2} \in \mathfrak{B}$. Let us put $\mathfrak{B}^{\prime}=\left\{\nabla_{2}: \nabla_{1} \times \nabla_{2} \in \mathfrak{B}\right\}$ and $\mathfrak{Z}(B)$ $=\left\{V_{1}: \nabla_{1} \times B \in \mathfrak{B}\right\}$ for $B \in \mathfrak{B}^{\prime}$. From Lemma (2.2) and Theorem (0.1) it follows that there exists a $\sigma$-discrete refinement $\mathfrak{F}(B)$ of $3(B)$. The family $\mathfrak{E}_{1}=\left\{F \times B: F \in \mathfrak{F}(B), B \in \mathfrak{B}^{\prime}\right\}$ has the required properties.

3. Subparacompactness in the Cartesian product of $\mathrm{GO}_{\delta}$-spaces. In the present section we shall investigate the following problem: under what conditions the product $\boldsymbol{P} X_{s}$ of $\mathbf{G O}_{\delta}$-spaces is subparacompact or hereditarily subparacompact?.

Let us recall that the Cartesian product of two $\mathrm{GO}_{\delta}$-spaces need not be paracompact (see [12], [9], [10] and [11]).

We shall consider three cases: $|S|>\aleph_{0},|S|=\aleph_{0}$ and $|S|<\aleph_{0}(|S|$ denotes cardinality of $S$ ).

Case 1. $|S|>\aleph_{0}$.

THEOREM 3.1. If the Cartesian product $\underset{s \in S}{\boldsymbol{P}} \boldsymbol{X}_{s}$ is subparacompact, where $X_{s}$ are $\mathrm{GO}_{\delta}$-spaces then the family $\left\{X_{s}\right\}_{s \in S}$ contains at most countably many non-compact spaces.

Theorem 3.1 is equivalent to the result obtained by the author and R. Engelking (see [1], Theorem 2), which states that the Cartesian product of $s_{1}$ copies of the discrete space of cardinality $s_{0}$ is not subparacompact.

Case 2. $|S|=\aleph_{0}$. 
The author does not know of any set of reasonably weak conditions which imply subparacompactness of $\underset{n=1}{\boldsymbol{P}} X_{n}$, where $X_{n}$ is a $\mathrm{GO}_{\delta}$-space. For hereditary subparacompactness we have

(3.2) THEOREM. If $X_{n}$ is a $\mathrm{GO}_{\delta}$ - space for $n=1,2, \ldots$, then the following conditions are equivalent:

(a) the Cartesian product $\underset{n=1}{\stackrel{\infty}{\boldsymbol{P}}} X_{n}$ is hereditarity countably subparacompact,

(b) the Cartesian product $\underset{n=1}{\mathbb{P}} X_{n}$ is hereditarity subparacompact.

The proof of Theorem 3.2 will be preceded by a lemma.

(3.3) Lemma. If the Cartesian product $\underset{i=1}{\boldsymbol{P}} X_{i}$, where $X_{i}$ is a $\mathrm{GO}_{\delta}$-space for $i=1,2, \ldots, n$, is hereditarily is countably subparacompact then it is hereditarily subparacompact.

Proof. We shall prove Lemma (3.3) by induction on $n$. If $n=1$ then Lemma (3.3) is obvious (see Theorem (0.1)). Let us assume that Lemma (3.3) holds for $n=k-1$. Let $G$ be an arbitrary open subset of the hereditarily countably subparacompact Cartesian product $X_{1} \times \ldots$ $\ldots \times X_{n}$ and $\mathfrak{U}$ its open covering. We can assume without loss of generality that if $U \in \mathfrak{U}$ then $U=U_{1} \times \ldots \times U_{k}$, where $U_{i}$ is an interval or a degenerate interval in $X_{i}$.

Let us notice that

(11) $\mid U \backslash\left\{\left[\operatorname{Int}_{M_{1}} U_{1}\right) \times U_{2} \times \ldots \times U_{k}\right] \cup\left[U_{1} \times\left(\operatorname{Int}_{M_{2}} U_{2}\right) \times U_{3} \times \ldots \times U_{k}\right] \cup$

$$
\left.\cup \ldots \cup\left[U_{1} \times \ldots \times U_{k-1} \times \operatorname{Int}_{M_{k}} U_{k}\right]\right\} \mid \leqslant 1,
$$

where $U \in \mathfrak{U}$ and $U=U_{1} \times \ldots \times U_{k}, M_{i}$ is a space associated with $X_{i}$, for $i=1,2, \ldots, k ; \mathfrak{U}$ is a covering of $G$ hence the set

$$
\begin{array}{r}
O=G \backslash\left(\cup \left\{\left[\left(\operatorname{Int}_{M_{1}} U_{1}\right) \times U_{2} \times \ldots \times U_{k}\right] \cup\left[U_{1} \times\left(\operatorname{Int}_{M_{2}} U_{2}\right) \times U_{3} \times \ldots \times U_{k}\right] \cup\right.\right. \\
\left.\cup \ldots \cup\left[U_{1} \times \ldots \times U_{k-1} \times\left(\operatorname{Int}_{M_{l k}} U_{k}\right)\right]: U_{1} \times \ldots \times U_{k} \in \mathfrak{L}\{\}\right)
\end{array}
$$

is closed and discrete in $G$.

We shall show that $C$ is a $G_{8}$-set. To do this let us denote by $V^{m}(x)$, for $m=1,2, \ldots$ and $x \in C$, an arbitrary open neighborhood of $x$ which satisfies the following conditions:

(12) $\quad \nabla^{m}(x)=V_{1}^{m}(x) \times \ldots \times V_{k}^{m}(x)$, where $V_{i}^{m}(x)$ is an interval or a degenerate interval in $X_{i}$ for $i=1,2, \ldots, k$.
(13) $\operatorname{diam} V_{i}^{m}(x)<1 / m$ (diam is defined with respect to a metric in $M_{i}$ ) for $i=1,2, \ldots, k$.

(14) $\delta\left\{V^{m}(x): m=1,2, \ldots, x \in C\right\}<\delta \mathfrak{U}\left({ }^{6}\right)$.

We shall show that $C=\bigcap_{m=1}^{\infty}\left(\cup\left\{V^{m}(x): x \in C\right\}\right) ;$ suppose on the contrary that there exist a point $y$ and a sequence of sets $\left\{V^{m}\left(x^{m}\right)\right\}_{m=1}^{\infty}$, where $x^{m} \in C$, such that

$$
\begin{gathered}
y \in X_{1} \times \ldots \times X_{k} \backslash C, \\
y \in V^{m}\left(x^{m}\right) \quad \text { for } \quad m=1,2, \ldots .
\end{gathered}
$$

We can assume without loss of generality that if $m \neq m^{\prime}$ then $x^{m} \neq x^{m^{\prime}}$ (see (15) and (16)). Let $p$ be the largest integer such that there are infinitely many points in the set $\left\{x^{m}\right\}_{m=1}^{\infty}$ which have $p$ coordinates in common with the point $y=\left(y_{1}, \ldots, y_{k}\right)$. We can assume, passing if necessary to a subsequence of $\left(x^{m}\right)_{m=1}^{\infty}$, that

and

$$
y_{i}=x_{i}^{m} \quad \text { for } \quad i=1,2, \ldots, p, \quad m=1,2, \ldots
$$$$
y_{i} \neq x_{i}^{m} \quad \text { for } \quad i=p+1, \ldots, k, m=1,2, \ldots,
$$

where $x^{m}=\left(x_{1}^{m}, \ldots, x_{k}^{m}\right)$. Let us notice that if $x=\left(x_{1}, \ldots, x_{k}\right) \in O$ then $\left\{x_{i}\right\}=\nabla_{i}^{m}(x) \backslash \operatorname{Int}_{M_{i}} V_{i}^{m}(x)$, hence the number $a=\inf \left(\varrho_{i}\left(y_{i}, X_{i} \backslash V_{i}^{1}\left(x^{1}\right)\right)\right.$ is positive. Let $m^{\prime}$ be a natural number such that $1 / m^{\prime}<a$. From the definition of $a,(13)$ and $(16)$ it follows that $V_{i}^{m^{\prime}}\left(x^{m^{\prime}}\right) \subset V_{i}^{1}\left(x^{1}\right)$ for $i=p+$ $+1, \ldots, k$, so we get $x^{m^{\prime}} \in V^{1}\left(x^{1}\right)$; the contradiction with (11) proves that $C$ is a $G_{\delta}$-set. Let

$$
\mathfrak{G}_{i}=\left\{U_{1} \times \ldots \times U_{i-1} \times\left(\operatorname{Int}_{M_{i}} U_{i}\right) \times U_{i+1} \times \ldots \times U_{k}: U_{1} \times \ldots \times U_{k} \in \mathfrak{H}\right\}
$$

and let $\mathfrak{B}_{i}=\bigcup_{n=1}^{\infty} \mathfrak{B}_{\text {in }}$ be a base in $M_{i}$, for $i=1,2, \ldots, k$, where $\mathfrak{B}_{\text {in }}$ is discrete in $M_{i}^{n=1}$. Denote by $\mathfrak{B}_{i}$ an arbitrary open refinement of $\mathfrak{G}_{i}$ such that if $\nabla \in \mathfrak{B}_{i}$ then $V=V_{1} \times \ldots \times V_{k}$, where $V_{i} \in \mathfrak{B}_{i}$ and $V_{j}$ is an interval or a degenerate interval in $X_{j}$ for $j \neq i$. Let

$$
\begin{aligned}
3_{i}(B)=\left\{V_{1} \times \ldots \times V_{i-1} \times V_{i+1} \times \ldots \times V_{k}: V_{1} \times \ldots\right. \\
\left.\ldots \times V_{i-1} \times B \times V_{i+1} \times \ldots \times V_{k} \in \mathfrak{B}_{i}\right\}
\end{aligned}
$$

and

$$
\mathfrak{B}_{i}^{\prime}=\left\{V_{i}: V_{1} \times \ldots \times V_{i} \times \ldots \times V_{k} \in \mathfrak{B}_{i}\right\}
$$

for $i=1,2, \ldots, k$. From the inductive assumption it follows that the set $\cup \mathcal{Z}_{i}(B)$ is subparacompact hence there exists a refinement $\mathfrak{\mho}_{i}(B)$

(') We shall write $\delta \mathfrak{U}<\delta \mathfrak{B}$, where $\mathfrak{U}$ and $\mathfrak{B}$ are families of subsets of $X$, if for every $U \in \mathfrak{I}$ there exists $V \in \mathfrak{B}$ such that $U \subset V$. 
of $3_{i}(B)$, which is $\sigma$-discrete in $\cup \mathcal{Z}_{i}(B)$ for $B \in B_{i}^{\prime}$ and $i=1,2, \ldots, k$. Let

$$
Y_{i n}=\bigcup\left\{V_{1} \times \ldots \times V_{k i} \in \mathfrak{B}_{i}: V_{i} \in \mathfrak{B}_{i n}^{\prime}\right\} \text {, where } \quad \mathfrak{B}_{i n}^{\prime}=\mathfrak{B}_{i}^{\prime} \cap \mathfrak{B}_{i n}
$$

for $i=1,2, \ldots, k$ and $n=1,2, \ldots$ The set $G$ is hereditary countably subparacompact, $G \backslash C$ is an $F_{\sigma}$-set and $\left\{Y_{i n}\right\}_{i=1}^{k_{i}} \underset{n=1}{\infty}$ is open, hence there exists a shrinking $\left\{D_{i n}\right\}_{i=1}^{k} \prod_{n=1}^{\infty}$ of $\left\{Y_{i n}\right\}_{i=1}^{l_{c}} \prod_{n=1}^{\infty}$ (this means that $D_{i n} \subset Y_{i n}$ and $\left.\bigcup_{i=1}^{k} \bigcup_{n=1}^{\infty} D_{i n}=G \backslash C\right)$ which consists of $F_{\sigma}$-sets in $G$. Thus $D_{i n}=\bigcup_{m=1}^{\infty} D_{i n m}$, where $D_{\text {inm }}$ is closed in $G$. Let us put

$$
\mathcal{D}=\bigcup_{i=1}^{k} \bigcup_{n=1}^{\infty} \bigcup_{m=1}^{\infty} \mathcal{D}_{i n m} \cup\{\{x\}\}_{x_{\epsilon} C}
$$

where

and

$$
D_{i n m}=\left\{D_{m}(F, B): F \in \Im_{i}(B), B \in \mathfrak{B}_{\text {in }}^{\prime}\right\}
$$

$$
D_{m}(F, B)=\left\{\left(x_{1}, \ldots, x_{k}\right) \in D_{i n m}:\left(x, \ldots, x_{i-1}, x_{i+1}, \ldots, x_{k}\right) \in F, x_{i} \in B\right\} .
$$

It is easy to verify that $\mathfrak{D}$ is a $\sigma$-discrete in $G$ refinement of $\mathfrak{H}$, hence our lemma is proved.

Proof of Theorem (3.2). The implication (b) $\Rightarrow(a)$ is obvious. Let us show that $(a) \Rightarrow(b)$. Let $\mathfrak{U}$ be an arbitrary open covering of an open subset $G$ of $\stackrel{\infty}{\boldsymbol{P}}_{n=1}$. We can assume without loss of generality that $\mathfrak{U}$ consists of sets of the form $U=\underset{n=1}{\stackrel{\infty}{\boldsymbol{P}}} B_{n}$, where $B_{n}$ is open in $X_{n}$ and the set $\left\{n: B_{n} \neq X_{n}\right\}$ is finite. Let

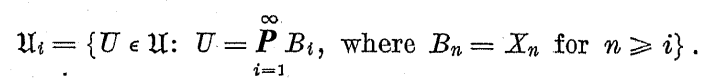

From Lemma (3.3) it follows that there exists a refinement $\mathfrak{F}_{i}$ of $\mathfrak{U}_{i}$ which is $\sigma$-discrete in $\bigcup \mathfrak{U}_{i}$. From the assumption that $G$ is countably subparacompact it follows that $\left\{\bigcup \mathfrak{H}_{i}\right\}_{i=1}^{\infty}$ has a shrinking $\left\{D_{i}\right\}_{i=1}^{\infty}$ such that $D_{i}=\bigcup_{n=1}^{\infty} D_{i n}$ and $D_{i n}$ is closed in $G$. The family $\Omega=\bigcup_{i=1}^{\infty} \bigcup_{n=1}^{\infty} \Omega_{i n}$, where $\mathfrak{K}_{i n}=\left\{K: K=D_{i n} \cap \mathbb{F}, \mathbb{F} \in \widetilde{F}_{i}\right\}$ is a refinement of $\mathfrak{U}$, which is $\sigma$-discrete in $G$, thus the proof is finished.

(3.4) Corollary (D. Lutzer [8]). The Cartesian product of $s_{0}$ copies of the Sorgenfrey line is hereditarily subparacompact.

Proof. In [5] it is proved that the Cartesian product of $\aleph_{0}$ copies of the Sorgenfrey line is perfect. To prove our Corollary it is sufficient to notice that every perfect space is hereditarily countably subparacompact (see Remark (1.2)).

Case 3. $|S|<\aleph_{0}$.

(3.5) THEOREM. If $X_{i}$ is a $\mathrm{GO}_{\delta}$-space such that $X_{i}=Y_{i} \cup Z_{i}$, where $\bar{Y}_{i}$ is perfect, $Z_{i} \subset X_{i} \backslash X_{i}^{d}$ and $\left|Z_{i}\right| \leqslant \aleph_{1}$, for $i=1,2$, then $X_{1} \times X_{2}$ is hereditarily subparacompact.

From Theorem (3.5) it follows that the Cartesian product $X_{1} \times X_{2}$, where $X_{1}$ and $X_{2}$ are perfect $\mathrm{GO}_{\delta}$-spaces, is hereditarily subparacompact. As Theorem (3.6) shows it is sufficient that one of the factors is perfect.

(3.6) THEOREM. If $X_{i}$ is a $\mathrm{GO}_{\delta}$-space for $i=1,2$ and $X_{1}$ is perfect then $X_{1} \times X_{2}$ is hereditarily subparacompact.

Theorems (3.5) and (3.6) will be proved simultaneously; the proof will be preceded by a lemma.

(3.7) LEMMA. If $X_{1}$ and $X_{2}$ satisfy assumptions of one of the above theorems, then the set $A=\left[\left(X_{1} \backslash X_{1}^{d}\right) \times X_{2}\right] \cup\left[X_{1} \times\left(X_{2} \backslash X_{2}^{d}\right)\right]$ is hereditarily subparacompact.

Proof. If $X_{1}$ is perfect then $A=A_{1} \cup A_{2}$, where $A_{1}=\left(X_{1} \backslash X_{1}^{d}\right) \times X_{2}$ and $A_{2}=A \backslash A_{1}$ are hereditarily subparacompact (Theorem 0.1 ) $F_{\sigma}$-sets, hence $A$ is hereditarily subparacompact.

Suppose now that $X_{1}$ and $X_{2}$ satisfy assumptions of Theorem 3.5. We can assume without loss of generality that $\left|Z_{1}\right|=\left|Z_{2}\right|=\aleph_{1}$ and $Z_{i}$ is disjoint to $Y_{i}$ for $i=1,2$ (if the set $Z_{i}$ is of cardinality $\aleph_{0}$ then $X_{i}$ is perfect (see Corollary (2.3)). Let $Z_{i}=\left\{x_{a}^{i}\right\}_{a<\omega_{1}}$ for $i=1,2$.

To finish the proof it is enough to show that the closed subset $F=A \backslash\left(U_{1} \cup U_{2}\right)$ of $A$, where $U_{1}=\left(Y_{1} \backslash X_{1}^{d}\right) \times X_{2}$ and $U_{2}=X_{1} \times\left(Y_{2} \backslash X_{2}^{d}\right)$, is hereditarily subparacompact. To do this we shall define disjoint $G_{\delta}$-sets $G_{1}$ and $G_{2}$ such that $G_{i} \supset B_{i}$ for $i=1,2$, where $B_{1}=X_{1}^{d} \times Z_{2}$ and $B_{2}$ $=Z_{1} \times X_{2}^{d}$. Let us put

$$
K_{a}=\left(\left\{x_{\alpha}^{1}\right\}\right) \times X_{2} \backslash \bigcup_{\beta<a} X_{1} \times\left\{x_{\beta}^{2}\right\} \quad \text { and } \quad L_{\alpha}=\left(X_{1} \times\left\{x_{\alpha}^{2}\right\}\right) \backslash \bigcup_{\beta \leqslant \alpha}\left(\left\{x_{\beta}^{1}\right\} \times X_{2}\right)
$$

for $\alpha<\omega_{1}$. It is easy to verify that the sets

$$
G_{1}=\left(\bigcup_{\alpha<\omega_{1}} L_{\alpha}\right) \cap F \quad \text { and } \quad G_{2}=\underset{\alpha<\omega_{1}}{\left(\bigcup K_{\alpha}\right) \cap F}
$$

have the required properties.

The sets $F_{i}=F \backslash G_{i}$ are hereditarily subparacompact $F_{\sigma}$-sets for $i=1,2$, therefore $F$ is hereditarily subparacompact and this completes the proof.

Proof of Theorems (3.5) and (3.6). Let $\mathfrak{U}$ be an arbitrary open covering of an open subset $G$ of $X_{1} \times X_{2}$. Let $\mathfrak{U}_{1}$ be an open family consisting of sets of the form $U=U_{1} \times U_{2}$, where $U_{i}$ is an interval in $X_{1}$ 
such that

$$
\cup \mathfrak{H}_{1} \supset G \cap\left(X_{1}^{d} \times X_{2}^{d}\right) \text { and } \delta \mathfrak{H}_{1}<\delta \mathfrak{H},
$$

From Lemma (2.4) it follows that there exists a $\sigma$-discrete covering $\mathfrak{E}$ of $H_{1} \cup H_{2}$, where

$$
H_{1}=\bigcup\left\{\left(\operatorname{Int}_{M_{1}} U_{1}\right) \times U_{2}: U_{1} \times U_{2} \in \mathfrak{H}_{1}\right\}
$$

and

$$
H_{2}=\bigcup\left\{U_{1} \times\left(\operatorname{Int}_{M} U_{2}\right): U_{1} \times U_{2} \in \mathfrak{H}_{1}\right\},
$$

such that $\delta \mathfrak{E}<\delta \mathfrak{U}_{1}$.

The argument used in the proof of Lemma (3.3) shows that the set $C=\bigcup \mathfrak{U}_{1} \backslash\left(H_{1} \cup H_{2}\right)$ is discrete in $\bigcup \mathfrak{H}_{1}$. From the properties of $\mathfrak{H}_{1}$ it follows that $C \subset G \cap\left(X_{1}^{d} \times X_{2}^{d}\right) \subset \cup \mathfrak{U}_{1}$, therefore $O$ is discrete and closed in $G$. The family $\mathfrak{E}_{1}=\mathbb{E} \cup\{\{x\}\}_{x \in C}$ is a $\sigma$-discrete in $G$ refinement of $\mathfrak{H}_{1}$ (to obtain this we used only the fact that $X_{i}$ are $\mathrm{GO}_{\delta}$-spaces). The set $F=G \backslash \cup \mathfrak{E}_{1}$ is closed in $G$ and $F \subset A$, hence from Lemma (3.7) it follows that there exists a $\sigma$-discrete closed refinement $\mathfrak{F}$ of $\mathfrak{U} \mid F$; therefore $\tilde{r}_{1} \cup \mathfrak{F}$ is a $\sigma$-discrete refinement of $\mathfrak{U}$ in $" G$.

(3.8) Corollary. If $X_{i}$ is a $\mathrm{GO}_{s^{-}}$space such that $\left|X_{i} \backslash X_{i}^{i}\right| \leqslant \aleph_{1}$ for $i=1,2$, then $X_{1} \times X_{2}$ is hereditarily subparacompact.

Corollary 3.8 follows from Corollary (2.3).

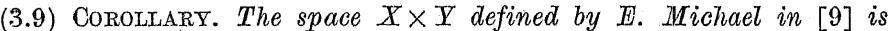
hereditarily subparacompact.

(3.10) Corollary. The Continum Hypothesis is equivalent to the following statement: if $X_{1}$ and $X_{2}$ are $\mathrm{GO}_{\delta}$-spaces and $X_{i}=X_{i} \cup Z_{i}$, where $Y_{i}$ is perfect, $Z_{i} \subset X_{i} \backslash X_{i}^{d}$ and $\left|Z_{i}\right| \leqslant 2^{N_{0}}$ for $i=1,2$, then the space $X_{1} \times$ $\times \bar{X}_{2}$ is hereditarily subparacompact.

Corollary (3.10) follows from Theorem (3.5) and the following

(3.11) Lemma. Under the assumption that $\aleph_{1}<2^{N_{0}}$ there exist Lindelöf $\mathrm{GO}_{\delta}^{- \text {spaces }} X_{1}, X_{2}$ such that the space $X_{1} \times X_{2}$ is not hereditarily subparacompact.

Proof. Let $X$ be a subset of the interval $I=[0,1]$ such that $|X|$ $=|I \backslash X|=2^{N_{0}}$ and both $X$ and $I \backslash X$ considered as subspaces of $I$ do not contain uncountable compact subsets (see [6], Theorem 1, p. 514). Let $L$ be an arbitrary subset of $I$ such that $|I \backslash L|=\aleph_{1}$.

Let us put $X=I_{X}$ and $X_{2}=I_{L}$ (see [4], Example 5.1.2). It is easy to verify that $X_{1}$ and $X_{2}$ are Lindelöf $\mathrm{GO}_{\delta}$-spaces.

We shall show that $A=\left(I_{X} \backslash X\right) \times I_{L} \cup I_{X} \times\left(I_{L} \backslash L\right)$ is not subparacompact. To do this, it is enough to prove that $B_{1}=X \times\left(I_{L} \backslash L\right)$ and $B_{2}=\left(I_{X} \backslash X\right) \times L$ cannot be separated by $G_{\delta}$-sets (see Remark (1.3)).
Suppose that $B_{i} \subset U_{i}$, for $i=1,2$, and $U_{i}$ are disjoint $G_{\delta}$-sets. From the properties of $X$ and the inequality, $2^{k_{0}}>s_{1}$ it follows that

and

Let us put

$$
\left|\left[\left(I_{X} \times\{x\}\right) \backslash U_{1}\right]\right| \leqslant \aleph_{0} \quad \text { for } \quad x \in I_{L} \backslash L
$$

$$
\left|\left[\left(\{x\} \times I_{L}\right) \backslash U_{2}\right]\right| \leqslant \aleph_{0} \text {. for } \quad x \in I_{X} \backslash X
$$

$$
B=\bigcap_{x \in(\Lambda \backslash L)} p\left[\left(I_{X} \times\{x\}\right) \frown U_{1}\right]
$$

where $p$ is the projection from $X_{1} \times X_{2}$ onto the first factor. From (19) and the inequality $2^{\aleph_{0}}>\aleph_{1}$ it follows that $B \backslash X \neq \varnothing$. Let $x$ be an arbitrary point of $B \backslash X$. It is easy to verify that $\{x\} \times\left(I_{L} \backslash L\right) \subset U_{1}$ but this contradicts $(20)$, because ${U_{1}}_{1} \cap \bar{U}_{2}=\emptyset\left({ }^{7}\right)$.

(3.12) CoRollaRY. The Continuum Hypothesis is equivalent to the following statement: if $X_{1}$ and $X_{2}$ are Lindelof $\mathrm{GO}_{\delta}$-spaces then the space $X_{1} \times X_{2}$ is hereditarily subparacompact.

To prove this fact it is enough to notice that $\left|M_{i}\right| \leqslant 2^{\mathrm{X}_{0}}$, where $M_{i}$ is associated with $X_{i}$ for $i=1,2$.

In Example (3.3) below we show that Theorem (3.5) does not hold for the Cartesian product of three spaces.

(3.3) EXAMPLE. We shall define perfect $\mathrm{GO}_{\delta}$-spaces $X_{1}, X_{2} \in \mathrm{GO}_{\delta}(I)$ such that the subspace $\Delta=\left\{(x, x): x \in I\right.$ of $X_{1} \times X_{2}$ will be homeomorphic to $I_{X}$ (see Lemma (3.11)).

Let $x$ be an arbitrary point of $I$. If $x \in X$ then the base at $x$ in $X_{1}$ and in $X_{2}$ is the same as in $I$, if $x \in X$ then the base at $x$ in $X_{1}$ consists of sets of the form $U=\{y \in I: x \leqslant y<a\}$ and the base at $x$ in $X_{2}$ of sets of the form $U=\{y \in I: a<y \leqslant x\}$. The space $X_{1} \times X_{2}$ has the required properties (see Lemma (2.3)).

If we assume that $2^{N_{0}}>N_{1}$ then $X_{1} \times X_{2} \times I_{L}$ is not hereditarily subparacompact because $X_{1} \times X_{2} \times I_{L} \supset I_{X} \times I_{L}$.

In the case of three spaces we have the following

(3.4) THEOREM. If $X_{i}$ is a perfect $\mathrm{GO}_{\delta}$-space for $i=1,2,3$, then the space $X_{1} \times X_{2} \times X_{3}$ is hereditarily subparacompact.

Proof. Let $G$ be an arbitrary open subset of the Cartesian product $X_{1} \times X_{2} \times X_{3}$ and $\mathfrak{U}$ its open covering. Let $\mathfrak{U}_{1}$ be an open family consisting of sets of the form $U=U_{1} \times U_{2} \times U_{3}$, where $U_{i}$ is an interval in $X_{i}$, for $i=1,2,3$, such that

$$
\cup \mathfrak{H}_{1} \supset G \cap\left(X_{1}^{d} \times X_{2}^{d} \times X_{3}^{d}\right) \text { and } \delta \mathfrak{H}_{1}<\delta \mathfrak{H} \text {, }
$$

(22) if $U_{1} \times U_{2} \times U_{3} \in \mathfrak{U}_{1}$, then $U_{i} \backslash\left(\operatorname{Int}_{M_{i}} U_{i}\right) \subseteq\left\{x_{i}\right\} \subset X_{i}^{d}$, where $M_{i}$ is associated with $X_{i}$ for $i=1,2,3$.

(?) R. Pol proved that the space $X_{1} \times X_{2}$ is subparacompact. 
From Lemma (2.4) it follows that there exists a $\sigma$-discrete covering $\mathfrak{E}$ of $H$, where

$$
\begin{array}{r}
H=\bigcup\left\{\left[U_{1} \times\left(\operatorname{Int}_{M_{2}} U_{2}\right) \times\left(\operatorname{Int}_{M_{3}} U_{3}\right)\right] \cup\left[\left(\operatorname{Int}_{M_{1}} U_{1}\right) \times U_{2} \times\left(\operatorname{Int}_{M_{8}} U_{3}\right)\right] \cup\right. \\
\left.\cup\left[\left(\operatorname{Int}_{M_{1}} U_{1}\right) \times\left(\operatorname{Int}_{M_{2}} U_{2}\right) \times U_{3}\right]: U_{1} \times U_{2} \times U_{3} \in \mathfrak{U}_{1}\right\}
\end{array}
$$

such that $\delta \mathfrak{E}<\delta \mathfrak{r}_{1}$.

Let us notice that the set

$$
\begin{aligned}
C=\bigcup \mathfrak{U}_{1} \backslash & \cup\left\{\left[\left(\operatorname{Int}_{M_{1}} U_{1}\right) \times U_{2} \times U_{3}\right] \cup\left[U_{1} \times\left(\operatorname{Int}_{M_{2}} U_{2}\right) \times U_{3}\right] \cup\right. \\
& \left.\left.\cup\left[U_{1} \times U_{2} \times\left(\operatorname{Int}_{M_{3}} U_{3}\right)\right]: U_{1} \times U_{2} \times U_{3} \in \mathfrak{H}_{1}\right\}\right)
\end{aligned}
$$

is discrete and closed in $\cup \mathfrak{U}_{1}$, similarily as the set $O$ from the proof of Lemma (3.3). From the properties of $\mathfrak{U}_{1}$ it follows that $O \subset G \cap$ $\cap\left(X_{1}^{d} \times X_{2}^{d} \times X_{3}^{d}\right) \subset \cup \mathfrak{U}_{1}$, therefore $C$ is discrete and closed in $G$.

Let us put

$$
\begin{aligned}
& D_{1}=\left\{V_{1}(U) \times D_{2}(U) \times D_{s}(U) \backslash H: \quad U \in \mathfrak{U}_{1}\right\} \\
& D_{2}=\left\{D_{1}(U) \times V_{2}(U) \times D_{s}(U) \backslash H: \quad U \in \mathfrak{U}_{1}\right\}
\end{aligned}
$$

and

$$
\mathfrak{D}_{3}=\left\{D_{1}(U) \times D_{2}(U) \times V_{3}(U) \backslash H: U \in \mathfrak{U}_{1}\right\}
$$

where $V_{i}(U)=\operatorname{Int}_{M_{i}} U_{i}$ and $D_{i}(U)=U_{i} \backslash \operatorname{Int}_{M_{i}}\left(U_{i}\right)$ for $U_{1} \times U_{2} \times U_{3}=U$. Notice that

$$
\left|D_{i}(U)\right| \leqslant 1 \text { and } U_{1} \times U_{2} \times U_{3} \backslash(C \cup H) \subset \bigcup_{i=1}^{3} \cup D_{i}
$$

therefore

$$
\bigcup \mathfrak{U}_{1} \backslash(O \cup H)=\bigcup_{i=1}^{3} \cup \mathfrak{D}_{i}
$$

Let $R_{1}$ be the equivalence relation in $D_{1}$ defined by the conditions

$$
\left(D_{1} \times D_{2} \times D_{3}\right) R_{1}\left(D_{1}^{\prime} \times D_{2}^{\prime} \times D_{3}^{\prime}\right) \quad \text { if } \quad D_{2}=D_{2}^{\prime} \text { and } D_{3}=D_{3}^{\prime} \text {. }
$$

Relations $R_{2}$ in $D_{2}$ and $R_{3}$ in $D_{3}$ are defined in analogous way.

Let us put

$\mathfrak{\Im}_{i}=\left\{F_{i}(D): D \in \mathfrak{D}_{i}\right\}, \quad$ where $\quad F_{i}(D)=\bigcup\left\{D^{\prime}: D^{\prime} R_{i} D\right\} \quad$ for

Let

$$
i=1,2,3 \text {. }
$$

$$
\mathfrak{F}_{i}^{n}=\left\{F_{i}^{n}(D)\right\}_{F_{i}(D) \in \Psi_{i}} \quad \text { for } \quad i=1,2,3 \text { and } n=1,2, \ldots
$$

where $F_{i}^{n}(D)=\left\{\left(x_{1}, x_{2}, x_{3}\right) \in F_{i}(D)\right.$ there exists $U_{1} \times U_{2} \times U_{3} \in \mathfrak{U}_{1}$ such that $\operatorname{diam} U_{j}>1 / n,\left\{x_{j}\right\}=U_{j} \backslash \operatorname{Int}_{M_{j}} U_{j}$ for $j \neq i$, and $\left.x_{i} \in \operatorname{Int}_{M_{i}} U_{i}\right\}$.
We shall show that the family $\mathfrak{F}_{i}^{n}$, for $i=1,2,3$ and $n=1,2, \ldots$ has the following properties:

(23) $\quad F_{i}^{n}(D)$ is an $F_{\sigma}$-set in $G$.

(24) $\mathfrak{F}_{i}^{n}$ is discrete in $\bigcup \mathfrak{F}_{i}$.

(25) $\mathfrak{\mho}_{i}^{n}$ is discrete in $\bigcup_{i=1}^{3} \cup \mathfrak{F}_{i}$.

(26) $\bigcup \mathfrak{F}_{i}^{n}$ is an $F_{\sigma}$-set in $\cup \mathfrak{U}_{1}$.

(27) If $F_{i}^{n}(D) \in \mathfrak{F}_{i}^{n}$ then there exists a $\sigma$-discrete covering $\mathfrak{U}_{i}^{n}(D)$ of $\mathfrak{F}_{i}^{n}(D)$ such that $\delta \mathfrak{U}_{i}^{n}(D)<\delta \mathfrak{U}$.

Ad (23). Any set $F_{i}^{n}(D) \in \mathfrak{F}_{i}^{n}$ is an $F_{\sigma}$-set in $G$ because it is an intersection of the $F_{\sigma}$-set $P=\bigcup\left\{P_{1} \times P_{2} \times P_{3}\right.$ there exists $U_{1} \times U_{2} \times U_{3} \in \mathfrak{U}_{1}$ such that $\operatorname{diam} U_{j}>1 / n, P_{j}=D_{j}=U_{j} \backslash \operatorname{Int}_{M_{j}}\left(U_{j}\right)=\left\{x_{j}\right\}$ for $j \neq i$ and $P_{i}=\operatorname{Int}_{M_{i}} U_{i}$, where $\left.D=D_{1} \times D_{2} \times D_{3}\right\}$, and the set $G \backslash H$, which is closed in $G$.

Ad (24). It is easy to verify that we do not change a set $D \in D_{i}$ if we put in its definition $D_{j}(U)=U_{j}$ instead of $U_{j} \backslash \operatorname{Int}_{M_{j}}\left(U_{j}\right)$ for $j \neq i$, therefore $F_{i}(D) \in \mathfrak{F}_{i}$ is open in $\bigcup_{i=1}^{3} \cup D_{i}$. The family $\mathfrak{F}_{i}$ consists of pairwise disjoint sets and $\delta \mathfrak{F}_{i}^{n}<\delta \mathfrak{F}_{i}$, hence $\mathfrak{F}_{i}^{n}$ is discrete in $\cup \mathfrak{F}_{i}$.

Ad (25). From (24) it follows that it is sufficient to show that for an arbitrary point $x \in \cup \mathfrak{F}_{j}$ and $j \neq i$ there exists an open neighbourhood of $x$ which intersects at most one element of $\mathfrak{F}_{i}^{n}$. We can assume without loss of generality that $i=1$ and $j=2$. Let us notice that the family $\mathfrak{F}_{2}$ is open in $\bigcup_{n=1}^{3} \cup \mathfrak{\mho}_{n}$ and if $F^{\prime}(D) \in \mathfrak{F}_{2}$ then $\left|p_{k}(F(D))\right|=1$, for $k=1,3$, where $p_{k}$ is the projection of $X_{1} \times X_{2} \times X_{3}$ onto $X_{k}$; therefore it is sufficient to show that the set $\left\{x_{1}\right\} \times X_{2} \times\left\{x_{3}\right\} \cap G \cap\left(\bigcup \mathfrak{F}_{1}^{n}\right)$, where $x=\left(x_{1}, x_{2}, x_{3}\right)$, is closed in $G$, and this can easily be verified.

Ad (26). From (23) and (25) it follows, that $\cup \mathfrak{F}_{i}^{n}$ is an $F_{\sigma}$-set in $\bigcup_{i=1}^{3} \cup \mathfrak{F}_{i}$. It is easy to see that

$$
\bigcup_{i=1}^{3} \cup \mathfrak{F}_{i}=\bigcup_{i=1}^{3} \cup \mathfrak{D}_{i}=\bigcup \mathfrak{U}_{1} \backslash(C \cup H)
$$

and $C \cup H$ is a $G_{\delta}$-set, hence $\cup \widetilde{F}_{i}^{n}$ is an $F_{\sigma}$-set in $\cup \mathfrak{U}_{1}$.

Ad (27). This is an obvious consequence of the definition of $\mathfrak{F}_{i}^{n}$ and metrizability of $M_{i}$.

From (25), (26), (27), the equality

$$
\bigcup_{i=1}^{3} \bigcup \mathfrak{F}_{i}=\bigcup_{i=1}^{3} \bigcup_{n=1}^{\infty} \bigcup \mathfrak{F}_{i}^{n}
$$


(28), and the properties of $\mathbb{E}$ and $O$ it follows that there exists a $\sigma$-discrete in $\bigcup \mathfrak{U}_{1}$ refinement $\mathfrak{F}_{1}$ of $\mathfrak{H}_{1}$. Notice that to obtain this refinement we used only the assumption that $X_{i}$ is a $\mathrm{GO}_{\delta}$-space for $i=1,2,3$.

Let us put $3=\varepsilon_{1} \mid\left(X_{1}^{d} \times X_{2}^{d} \times X_{3}^{d}\right) \cap G$. From (21) it follows that 3 is a $\sigma$-discrete, in $G$, covering of $G \cap\left(X_{1}^{d} \times X_{2}^{d} \times X_{3}^{d}\right)$ and $\delta 3<\delta \mathfrak{H}$.

We shall show that $T=G \backslash\left(X_{1}^{d} \times X_{2}^{d} \times X_{3}^{d}\right)$ is a subparacompact $E_{a}$-set in $G$. To do this let us put

and

$$
\begin{aligned}
& A_{1}=\left[\left(X_{1} \backslash X_{1}^{d}\right) \times X_{2} \times X_{3}\right] \cap G, \\
& A_{2}=\left[X_{1} \times\left(X_{2} \backslash X_{2}^{d}\right) \times X_{3}\right] \cap G
\end{aligned}
$$

$$
A_{3}=\left[X_{1} \times X_{2} \times\left(X_{3} \backslash X_{3}^{d}\right)\right] \cap G
$$

The space $X_{i}$ is perfect, hence $A_{i}$ is an $F_{\sigma}$-set in $G$ and from Theorem (3.5) and the equality $T=A_{1} \cup A_{2} \cup A_{3}$ it follows that $T$ is a subparacompact $F_{\sigma}$-set in $G$.

Let $\mathfrak{I}$ be a $\sigma$-discrete in $G$ covering of $T$ such that $\delta \mathfrak{I}<\delta \mathfrak{U}$. The family $\mathfrak{I} \cup \mathfrak{Z}$ is a $\sigma$-discrete refinement of $\mathfrak{U}$ and the proof is finished.

By using Corollary (2.3) we infer

(3.5) CoRollary. If $X_{i}$ is a $\mathrm{GO}_{\delta}$-space without isolated points, for $i=1,2,3$, then the space $X_{1} \times X_{2} \times X_{3}$ is hereditarity subparacompact.

Applying the method from Example (3.3), we easily notice that Theorem 3.4 does not hold for four spaces.

(3.16) THEOREM. If $X_{i}$ is a $\mathrm{GO}_{\delta}$-space, for $i=1,2,3,4$, then the following conditions are equivalent:

(a) the Cartesian product $X_{1} \times X_{2} \times X_{3} \times X_{4}$ is finitely subparacompact (hereditarily finitely subparacompact),

(b) the Cartesian product $\bar{X}_{1} \times X_{2} \times X_{3} \times X_{4}$ is subparacompact (hereditarily subparacompact).

The proof of Theorem (3.6) will be preceded by two lemmas.

(3.17) LEMMA. If $X_{i}$ is a $\mathrm{GO}_{\delta}$-space, for $i=1,2$, and the Cartesian product $X_{1} \times X_{2}$ is finitely subparacompact, then $X_{1} \times X_{2}$ is subparacompact.

Proof. Let $\mathfrak{U}$ be an arbitrary open covering of $X_{1} \times X_{2}$. Wo obtain, in the same way as in the proof of Theorem (3.5) and Theorem (3.6), a $\sigma$-discrete family $\mathfrak{E}_{1}$ such that $\delta \mathfrak{F}_{1}<\delta \mathfrak{H}, \cup \mathfrak{E}_{1} \supset X_{1}^{d} \times X_{2}^{c l}$ and the set $\cup \mathcal{E}_{1}$ is open in $X_{1} \times X_{2}$. From the assumption that $X_{1} \times X_{2}$ is finitely subparacompact it follows that there exist $F_{\sigma}$-sets $F_{1}, F_{2}$ and $F_{3}$ satisfying the following conditions

$$
\begin{gathered}
F_{1} \cup F_{2} \cup F_{3}=X_{1} \times X_{2}, \\
F_{1} \subset\left(X_{1} \backslash X_{1}^{d}\right) \times X_{2},
\end{gathered}
$$

$$
\begin{gathered}
F_{2} \subset X_{1} \times\left(X_{2} \backslash X_{2}^{d}\right) \\
F_{3} \subset \cup \mathscr{F}_{1}
\end{gathered}
$$

The sets $F_{1}$ and $F_{2}$ are subparacompact and the family $\mathbb{F}_{1} \mid F_{3}$ is $\sigma$-discrete, hence $X_{1} \times X_{2}$ is subparacompact.

(3.18) Lemana. If $X_{i}$ is a $\mathrm{GO}_{\delta}$-space, for $i=1,2,3$, and the Cartesian product $X_{1} \times X_{2} \times X_{3}$ is finitely subparacompact then $X_{1} \times X_{2} \times X_{3}$ is subparacompact.

Proof. Let $\mathfrak{U}$ be an arbitrary open covering of $X_{1} \times X_{2} \times X_{3}$. In the same way as in the proof of Theorem (3.4) we obtain a family $\mathfrak{F}_{1}$ which is $\sigma$-discrete in $\bigcup \mathfrak{E}_{1}$ such that $\delta \mathfrak{F}_{1}<\delta \mathfrak{U}, \cup \mathfrak{E}_{1} \supset X_{1}^{d} \times X_{2}^{d} \times X_{3}^{d}$ and the set $U \tilde{F}_{1}$ is open in $X_{1} \times X_{2} \times X_{3}$. From the assumption that $X_{1} \times$ $\times X_{2} \times X_{3}$ is finitely subparacompact it follows that there exists $F_{\sigma}$-sets $F_{1}, F_{2}, F_{3}$ and $F_{4}$ which satisfy the following conditions:

$$
\begin{gathered}
F_{1} \cup F_{2} \cup F_{3} \cup F_{4}=X_{1} \times X_{2} \times X_{3}, \\
F_{1} \subset\left(X_{1} \backslash X_{1}^{d}\right) \times X_{2} \times X_{3}, \\
F_{2} \subset X_{1} \times\left(X_{2} \backslash X_{2}^{d}\right) \times X_{3}, \\
F_{3} \subset X_{1} \times X_{2} \times\left(X_{3} \backslash X_{3}^{d}\right) . \\
F_{4} \subset \cup \mathfrak{E}_{1} .
\end{gathered}
$$

The sets $F_{1}, F_{2}, F_{3}$ are subparacompact (see Lemma (3.7)) and the family $\tilde{E}_{1} \mid F_{4 n}$, where $F_{4 n}$ are closed subsets of $X_{1} \times \dot{X}_{2} \times X_{3}$ such that $\bigcup_{n=1}^{\infty} F_{4 n}=F_{4}$, is discrete, hence $X_{1} \times X_{2} \times X_{3}$ is subparacompact.

Proof of Theorem (3.6). The implication (b) $\Rightarrow(a)$ is obvious; to prove Theorem (3.6) it is sufficient to show (a) $\Rightarrow(b)$.

Let $\mathfrak{U}$ be an arbitrary open covering of $X=X_{1} \times X_{2} \times X_{3} \times X_{4}$. Let $\mathfrak{U}_{1}$ be an open family consisting of sets of the form $U=U_{1} \times U_{2} \times$ $\times U_{3} \times U_{4}$, where $U_{i}$ is an interval in $X_{i}$, for $i=1,2,3,4$, such that

$(40)$ if $U_{1} \times U_{2} \times U_{3} \times U_{4} \in \mathfrak{U}_{1}$, then $U_{i} \backslash \operatorname{Int}_{M_{i}} U_{i} \subset\left\{x_{i}\right\} \subseteq X_{i}^{d}$.

Let $\mathfrak{I}_{i}=\left\{T \subset T_{4}:|T|=i\right\}$, where $i=1,2,3,4$ and $T_{4}=\{1,2,3,4\}^{\bullet}$ Let us put for $T \in \mathfrak{I}_{1}$ :

$$
\begin{array}{r}
\Re_{1}(T)=\left\{D_{1} \times D_{2} \times D_{3} \times D_{4}: D_{t}=U_{t} \text { for } t \in T \text { and } D_{m}=\operatorname{Int}_{M_{m}} U_{m}\right. \\
\text { for } \left.m \in T_{4} \backslash T, \text { where } U_{1} \times U_{2} \times U_{3} \times U_{4} \in \mathfrak{U}_{1}\right\} .
\end{array}
$$

Suppose we have already defined $\Omega_{j}(T)$ for $j<i$ and $T \in \mathfrak{I}_{j}$; let us put 
for $T \in \mathfrak{I}_{i}$

$\Omega_{i}(T)=\left\{D_{1} \times D_{2} \times D_{3} \times D_{4} \backslash \bigcup_{j<i} \bigcup_{T^{\prime} \in \mathfrak{X}_{j}} \cup \Re_{j}\left(T^{\prime}\right): D_{t}=U_{t} \backslash\right.$ Int $_{M X_{t}} U_{t}$ for $t \in T$

and $D_{m}=\operatorname{Int}_{M_{m}} U_{m}$ for $m \in T_{\mathbf{4}} \backslash T$, where $\left.U_{1} \times U_{2} \times U_{3} \times U_{\mathbf{4}} \in \mathfrak{L}_{1}\right\}$.

The argument as in the proof of Lemma (3.3) shows that $\mathfrak{\Re}_{4}\left(T_{4}\right)$ is discrete in $\bigcup \mathfrak{H}_{1}$. From (39) and (40) it follows that $\cup \mathfrak{R}_{4}^{\prime}\left(T_{4}\right) \subset X_{1}^{d} \times X_{2}^{d} \times$ $\times X_{3}^{d} \times X_{4}^{d} \subset \cup \mathfrak{U}_{1}$, hence $\Omega_{4}\left(T_{4}\right)$ is discrete in $X_{1} \times X_{2} \times X_{3} \times X_{4}$.

Applying Lemma (2.4) we find a covering $\mathbb{E}$ of $\bigcup_{T \in \tau_{1}} \cup \Omega_{1}(T)$ which is $\sigma$-discrete in $X_{1} \times X_{2} \times X_{3} \times X_{4}$ and such that $\delta \mathfrak{E}<\delta \mathfrak{U}$.

We define the equivalence relation $R(i, T)$ in $\Omega_{i}(T)$ where $T \in \mathfrak{I}_{i}$ and $i=2,3$, assuming

$$
K R(i, T) K^{\prime} \quad \text { if } \quad p\left(K^{\prime}\right)=p_{j}(K) \quad \text { for } \quad j \in T,
$$

where $p_{j}$ is the projection of $X_{1} \times X_{2} \times X_{3} \times X_{4}$ onto $X_{j}$. Let $\mathfrak{F}(i, T)$ $=\left\{F^{\prime}(K): K \in \mathfrak{\Re}_{i}(T)\right\}$ for $T \in \mathfrak{I}_{i}$, where $F^{\prime}(K)=\bigcup\left\{K^{\prime}: K^{\prime} R\left(i, T^{\prime}\right) K\right\}$.

We shall show that the family $\mathfrak{F}(i, T)$, for $T \in \mathfrak{I}_{i}$ and $i=2,3$, has the following properties:

(41) elements of $\mathfrak{F}(i, T)$ are open in $\underset{T \in \mathbb{T}_{i}}{\bigcup} \cup \mathfrak{F}(i, T)$,

(42) $\mathfrak{F}(i, T)$ is discrete in the $\cup \mathfrak{F}(i, T)$,

(43) $\bigcup \mathscr{F}(3, T)$ is a $G_{\delta}$-set in $X_{1} \times X_{2} \times X_{3} \times X_{4}$,

(44) $\bigcup \cup \mathfrak{F}(i, T)$ is an $F_{\sigma}$-set in $\bigcup \mathfrak{H}_{1}$.

(45) if $F(K) \in \mathfrak{F}(i, T)$ then there exists a $\sigma$-discrete covering $\mathfrak{X}(F(K)$ of $F^{\prime}(K)$ such that $\delta \mathfrak{U}(F(K))<\delta \mathfrak{U}_{1}$.

Ad (41). It is easy to see that we do not change a set $F(K) \in \mathfrak{F}(i, T)$ if we put in its definition $D_{t}=U_{t}$ instead of $U_{t} \backslash \operatorname{Int}_{M_{t}}\left(U_{t}\right)$ for $t \in T$, hence $F(K) \in \mathfrak{F}(i, T)$ is open in $\bigcup_{T \in \mathfrak{T}_{i}} \cup \mathfrak{F}(i, T)$ as the union of open subsets of $\bigcup_{T \in \mathcal{T}_{i}} \cup \mathfrak{F}(i, T)$.

Ad (42). The elements of $\mathfrak{F}(i, T)$ are pairwise disjoint hence from (4:1) it follows that $\mathfrak{F}(i, T)$ is discrete in $\bigcup \mathfrak{F}(i, T)$.

Ad (43). From (39) and (40), the definition of $\mathfrak{F}(3 ; T)$, and Lemma (2.2) it follows that the elements of $\mathbb{F}(3, T)$ are of the form

(46) $\quad F(K)=P_{1} \times P_{2} \times P_{3} \times P_{4}$, where $\left|P_{t}\right|=1$ for $t \in T$ and $P_{m}$ is a $G_{\delta}$-set in $M_{m}$ for $m \in T_{4} \backslash T$.

We can assume without loss of generality that $T=\{1,2,3\}$ and $m=T_{4} \backslash T=\{4\}$.
Let $\nabla^{n}(x)$ be an arbitrary neighborhood of $x=\left(x_{1}, x_{2}, x_{3}, x_{4}\right)$ $\epsilon \cup \mathfrak{F}(3, T)$ satisfying the following conditions for $n=1,2,3, \ldots$

(47) $\quad V^{n}(x)=V_{1}^{n}(x) \times V_{2}^{n}(x) \times V_{3}^{n}(x) \times V_{4}^{n}(x)$, where $\nabla_{i}^{n}(x)$ is an interval in $X_{i}$ for $i=1,2,3$ and $V_{4}^{n}(x)$ is an open subset of $M_{4}$,

(50) if $x \in F(K)=P_{1} \times P_{2} \times P_{3} \times P_{4} \in \mathfrak{F}(3, T)$, then $V_{4}^{n}(x) \subset G_{n}$, where $G_{n}$ is open subset of $M_{4}$ such that $\bigcap_{n=1}^{\infty} G_{n}=P_{4}$.

Let us assume that the equality

$$
\mathfrak{F}(3, T)=\bigcap_{n=1}^{\infty}\left(\bigcup\left\{\nabla^{n}(x): x \epsilon \bigcup \mathfrak{F}(3, T)\right\}\right)
$$

does not hold. Then there exist a point $y$ and a sequence of sets $\left(\left.V^{n}\left(x^{n}\right)\right|_{n=1} ^{\infty}\right.$, where $x^{n} \in \bigcup \mathfrak{F}(3, T)$, such that

$$
\begin{gathered}
y \in X_{1} \times X_{2} \times X_{3} \times X_{4} \backslash(\bigcup F(3, T)), \\
y \in V^{n}\left(x^{n}\right) \text { for } n=1,2, \ldots
\end{gathered}
$$

From (51), (52), (46) and from the properties of the sets $V^{n}(x)$ it follows that there exists an infinite subset of $\left\{x^{n}\right\}_{n=1}^{\infty}$, such that its intersection with each element of $\mathfrak{F}(3, T)$ is of cardinality $\leqslant 1$. We can assume without loss of generality that

(53) $\quad\left|F(K) \cap\left\{x^{1}, x^{2}, \ldots\right\}\right| \leqslant 1 \quad$ for $\quad F(K) \in \mathbb{F}(3, T)$ and $x^{n} \neq x^{m}$ if

$$
n \neq m \text {. }
$$

Let $k$ be the maximal integer such that there exist infinitely many points in $\left\{x^{n}\right\}_{n=1}^{\infty}$ which have $k$ coordinates among the first three coordinates in common with the point $y=\left(y_{1}, y_{2}, y_{3}, y_{4}\right)$. We can assume, passing if necessary to a subsequence of $\left(x^{n}\right)_{n=1}^{\infty}$, that for $n=1,2, \ldots$ $y_{i}=x_{i}^{n}$ if $i \leqslant k$ and $y_{i} \neq x_{i}^{n}$ if $k<i \leqslant 4$.

Let us notice that if $x=\left(x_{1}, x_{2}, x_{3}, x_{4}\right) \in \bigcup \mathfrak{F}(3, T)$, where $T=\{1,2,3\}$, then $\left\{x_{i}\right\}=V_{i}^{n}(x) \backslash \operatorname{Int}_{M_{i}} V_{i}^{n}(x)$ for $i=1,2,3$, so $a=\inf _{k<i} \varrho_{i}\left(y_{i}, X_{i} \backslash V_{i}^{1}\left(x^{1}\right)\right)$ $>0$. Let $n$ be a natural number such that $1 / n<a$. From the definition of $a,(48)$ and (52) it follows that $V_{i}^{n}\left(x^{n}\right) \subset V_{i}^{1}\left(x^{1}\right)$ for $k<i \leqslant 4$; as $x_{i}^{n}=x_{i}^{1}$ for $i \leqslant k$, hence $x^{n} \in V^{1}\left(x^{1}\right)$, but it is impossible because

$$
V^{1}\left(x^{1}\right) \cap\left\{x^{n}: n=2,3, \ldots\right\}=\emptyset,
$$

therefore $\bigcup \mathfrak{F}(3, T)$ is a $G_{\delta}$-set.

Ad (44). It is easy to verify that

$\bigcup_{T \in \mathfrak{I}_{3}} \cup \mathfrak{F}(2, T)=\left(\cup \mathfrak{X}_{1}\right) \backslash\left(\bigcup_{T \in \mathfrak{T}_{1}} \cup \mathfrak{H}(1, T) \cup \bigcup_{T \in \mathfrak{T}_{3}} \cup \mathfrak{F}(3, T) \cup \cup \mathfrak{R}\left(4, T_{4}\right)\right)$, 
and

$$
\left.\bigcup_{T \in \mathfrak{I}_{3}} \cup \mathfrak{F}(3, T)=\bigcup \mathfrak{H}_{1} \backslash \bigcup_{i=1}^{2} \bigcup_{T \in \mathfrak{T}_{i}} \cup \mathfrak{R}(i, T) \cup \bigcup \mathfrak{K}\left(4, T_{4}\right)\right)
$$

Let us notice that the sets $\bigcup_{T \in \mathfrak{I}_{1}} \cup \mathfrak{R}(1, T)$ and $\bigcup_{i=1}^{2} \bigcup_{T \in \mathfrak{I}_{i}} \cup \Re(i, T)$ are open, and $\cup \mathfrak{F}(3, T)$ and $\Re\left(4, T_{4}\right)$ are $G_{\delta}$-sets; therefore (44) hold.s.

Ad (45). This is an obvious consequence of metrizability of $M_{i}$ and the definition of $F(K)$.

The Cartesian product $X_{1} \times X_{2} \times X_{3} \times X_{4}$ is finitely subparacompact, therefore there exist $F_{\sigma}$-sets $F_{1}, F_{2}, F_{3}, F_{4}$, and $F_{5}$ such that

$$
\begin{aligned}
& \bigcup_{i=1}^{5} F_{i}=X_{1} \times X_{2} \times X_{3} \times X_{4}, \\
& F_{1} \subset\left(X_{1} \backslash X_{1}^{d}\right) \times X_{2} \times X_{3} \times X_{4}, \\
& F_{2} \subset X_{1} \times\left(X_{2} \backslash X_{2}^{d}\right) \times X_{3} \times X_{4}, \\
& F_{3} \subset X_{1} \times X_{2} \times\left(X_{3} \backslash X_{3}^{d}\right) \times X_{4}, \\
& F_{4} \subset X_{1} \times X_{2} \times X_{3} \times\left(X_{4} \backslash X_{4}^{d}\right),
\end{aligned}
$$

$$
F_{5} \subset \cup \mathfrak{U}_{1} \text {. }
$$

From the fact that finite subparacompactness is hereditary with respect to $F_{\sigma}$-sets and from (44), (41), (42) and (45) it follows that there exists a $\sigma$-discrete family $\mathfrak{E}(i, T)$ for $T \in \mathfrak{I}_{i}$ such that

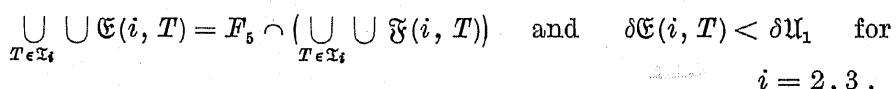

Let

$$
\mathfrak{E}_{1}=\underset{T \in \mathfrak{I}_{2}}{\bigcup l \mathbb{E}}(2, T) \cup \bigcup_{T \in \mathfrak{I}_{3}} \mathfrak{E}(3, T) \cup \mathfrak{E} \cup\{x\}_{x \in \mathcal{K}_{4}\left(4, T_{4}\right)} .
$$

It is easy to see that $E_{1}$ is a $\sigma$-discrete refinement of $\mathfrak{U}_{1} \mid F_{5}$ and this completes the proof because $F_{i}$ for $i \leqslant 4$ is subparacompact and an $F_{\sigma}$-set (see Lemma 3.18).

The proof of the part in brackets is similar; it is sufficient to replace the product $X_{1} \times X_{2} \times X_{3} \times X_{4}$ by its arbitrary open subset.

The author does not know whether Theorem (3.16) holds for the product of five spaces.

(3.19) THeorem. If the Cartesian product $X_{1} \times \ldots \times X_{n}$ of $\mathrm{GO}_{\delta}$-spaces is collectionwise normal then it is subparacompact, and a fortiori paracompact.

Proof. Let $\mathfrak{U}$ be an arbitrary open covering of $X=X_{1} \times \ldots \times X_{n}$. We can assume without loss of generality that $\mathfrak{U}$ consists of sets of the form $U=U_{1} \times \ldots \times U_{n}$, where $U_{i}$ is an interval or a degenerate interval in $X_{i}$, for $i=1,2, \ldots, n$.
Let $\mathfrak{I}_{i}=\left\{T \subset T_{n}:|T|=i\right\}$, where $i=1,2, \ldots, n, T_{n}=\{1,2, \ldots, n\}$ and $T_{0}=\{\varnothing\}$.

Let $\Omega_{0}(\varnothing)=\left\{\left(\operatorname{Int}_{M_{1}} U_{1}\right) \times\left(\operatorname{Int}_{M_{2}} U_{2}\right) \times \ldots \times \operatorname{Int}_{M_{n}}\left(U_{n}\right): U_{1} \times \ldots \times U_{n} \in \mathfrak{U}\right\}$. Suppose we have already defined $\Omega_{j}(T)$ for $j<i$ and put for $T \in \mathfrak{I}_{i}$

$$
\begin{array}{r}
\mathfrak{K}_{i}(T)=\left\{D_{1} \times \ldots \times D_{n} \backslash\left(\bigcup_{j<i} \bigcup_{T^{\prime} \epsilon \mathfrak{T}_{1}} \cup \Re_{j}\left(T^{\prime}\right)\right): D_{t}=U_{t} \backslash \operatorname{Int}_{M_{t}} U_{t} \text { for } t \in T,\right. \\
\left.D_{j}=\operatorname{Int}_{M_{j}} U_{j} \text { for } j \in T_{n} \backslash T, \text { where } U_{1} \times \ldots \times U_{n} \in \mathfrak{U}\right\} .
\end{array}
$$

We define a relation $R(i, T)$ in $\mathcal{K}_{i}(T)$, where $T \in \mathfrak{I}_{i}$ and $i=1,2, \ldots, n$, assuming $K R(i, T) K^{\prime}$ if $p_{j}\left(K^{\prime}\right)=p_{j}(K)$ for $j \in T$, where $p_{j}$ is the projection from $\stackrel{\boldsymbol{P}}{\boldsymbol{P}}_{i=1} X_{i}$ onto $X_{j}$.

Let $\mathfrak{F}(i, T)=\left\{F(K): K \in \Omega_{i}(T)\right\}$, where $F(K)=\bigcup\left\{K^{\prime}: K^{\prime} R(i, T) K\right\}$.

It is easy to verify that $\mathfrak{F}(i, T)$ for $T \in \mathfrak{I}_{i}$ and $i=1,2, \ldots, n$ has the following properties:

(60) $\mathfrak{F}\left(n, T_{n}\right)$ is discrete,

(61) $\mathfrak{F}(i, T)$ is discrete in $\bigcup \mathfrak{F}(i, T)$ and consists of sets open in $\bigcup_{T \in \mathfrak{I}_{i}} \cup \mathfrak{F}(i, T)$

(62) $\bigcup_{T \in \mathfrak{T}_{i}} \cup \mathfrak{F}(i, T)$ is a closed subset of $\bigcup_{j=1}^{i} \bigcup_{T \in \mathfrak{T}_{j}} \cup \mathfrak{F}(j, T)$,

(63) $\bigcup_{i=1}^{n} \bigcup_{T \in \mathfrak{I}_{i}} \cup \mathfrak{F}(i, T) \cup \cup \Re_{0}(\varnothing)=X$.

(64) if $F(K) \in \mathfrak{F}(i, T)$, then there exists a $\sigma$-discrete open family $\mathfrak{U}(F(K))$ such that $\bigcup \mathfrak{U}(F(K)) \supset F(K)$ and $\delta \mathfrak{U}(F(K))<\delta \mathfrak{U}$.

The family $\Omega_{0}(\varnothing)$ consists of open subsets of $M_{1} \times \ldots \times M_{n}$, hence the proof will be finished if we define for $i=1,2, \ldots, n$ a $\sigma$-discrete open family $\mathfrak{B}_{i}$ such that $\delta \mathfrak{B}_{i}<\delta \mathfrak{U}$ and $\cup \mathfrak{D}_{i} \supset \underset{T \in \mathfrak{I}_{i}}{\bigcup} \mathfrak{W}(i, T)$.

From (60) and collectionwise normality of $X$ it follows that there exists $\mathfrak{V}_{n}$ which satisfies both required properties.

Suppose we have already defined $\mathfrak{B}_{j}$ for $j<i$. Let

$$
Z=\left(\left(\bigcup_{T \in \mathfrak{T}_{i}} \cup \mathfrak{F}(i, T)\right) \backslash\left(\bigcup \mathfrak{R}_{0}(\emptyset) \cup \cup \mathfrak{D}_{n} \cup \ldots \cup \cup \mathfrak{B}_{i+1}\right) .\right.
$$

It is easy to verify that $Z$ is closed (see (63) and (62)), therefore from the normality of $X$ and (61) it follows that there exists a family $3=\{Z(T)$ : $\left.T \in \mathfrak{I}_{i}\right\}$ of closed sets such that

$$
Z(T) \subset \bigcup \mathfrak{F}(i, T) \backslash\left(\mathfrak{\Omega}_{0}(\varnothing) \cup\left(\bigcup \mathfrak{B}_{n} \cup \ldots \cup \cup \mathfrak{B}_{i+1}\right)\right.
$$

and

(66)

$$
\cup 3=Z
$$


From (61), (64), collectionwise normality of $X$, and (65) it follows that there exists an open $\sigma$-discrete family $\mathfrak{B}(T)$ for $T \in \mathfrak{I}_{i}$ such that $\delta \mathfrak{B}(T)<\delta \mathfrak{U}$ and $\cup \mathfrak{B}(T) \supset Z(T)$.

Applying (66) we see that $\mathfrak{B}_{i}=\mathfrak{B}_{n} \cup \ldots \cup \mathfrak{B}_{i+1} \cup \Omega_{0}(\varnothing) \cup \bigcup_{T \in \mathfrak{I}_{i}} \mathfrak{B}(T)$ has the required properties, and the proof is finished.

Let us notice that using similar arguments we can prove that if $X$ is hereditarily collectionwise normal, then $X$ is hereditarily paracompact.

(3.20) CoRoLlaRY. Let us assume that $2^{\mathrm{N}_{0}}<2^{\mathrm{K}_{1}}$. If $X_{i}$ is a hereditarily Lindelöf $\mathrm{GO}_{\diamond}$-space for $i=1,2, \ldots, n$ then the following conditions are equivalent:

(a) the Cartesian product $X_{1} \times \ldots \times X_{n}$ is normal (hereditarily normal),

(b) the Cartesian product $X_{1} \times \ldots \times X_{n}$ has the Lindelöf property (is hereditarily Lindelöf).

Proof. The implication $(b) \Rightarrow(a)$ is obvious; to prove Corollary (3.20) it is sufficient to show that (a) $\Rightarrow$ (b).

Let us notice that if $U$ is an open subset of $X_{i}$ and $U \cap X_{i}^{d} \neq \varnothing$ then $\operatorname{Int}_{M_{i}} U_{i} \neq \emptyset$, hence from the inequalities $w\left(M_{i}\right) \leqslant \kappa_{0}$, where $w\left(M_{i}\right)$ denotes the weight of $M_{i}$, and $\left|M_{i} \backslash M_{i}^{d}\right| \leqslant \aleph_{0}$ it follows that $X_{1} \times \ldots \times X_{n}$ is separable as a finite product of separable spaces. By the standard arguments (see [4], Example 1.52 or 2.1.2) we can prove that $X$ is collectionwise normal.

(3.21) COROLLARY. If $X_{i}$ is a hereditarily Lindelöf $\mathrm{GO}_{\delta}$-space for $i=1,2, \ldots, n$ then the equivalence of the conditions (a) and (b) of Corollary (3.20) is independent of the usual axioms of set theory.

The proof follows from Corollary (3.20) and from the fact that it is consistent with the usual axioms of set theory to assume that there exists a hereditarily Lindelöf $\mathrm{GO}_{\delta}$-space $X$ such that the Cartesian product $X \times X$ is hereditarily normal but not paracompact (see [11]).

(3.22) Conollary. Let us assume that $2^{\mathrm{N}_{0}}<2^{\mathrm{K}_{1}}$. If $X_{i}$ is a Lindelöf $\mathrm{GO}_{\delta}$-space for $i=1,2, \ldots, n$ then following conditions are equivalent:

(a) the Cartesian product $X_{1} \times \ldots \times X_{n}$ is normal (hereditarily normal),

(b) the Cartesian product $X_{1} \times \ldots \times X_{n}$ is paracompact (hereditarily paracompact).

Proof. The implication (b) $\Rightarrow(a)$ is obvious; to prove Corollary (3.22) it is sufficient to show that (a) $\Rightarrow(b)$.

We shall prove the implication $(a) \Rightarrow(b)$ by induction on $n$. For $n=1$ the implication is trivial. Let us assume that the implication $(\mathrm{a}) \Rightarrow(\mathrm{b})$ holds if $n=k-1$. Let $\mathfrak{U}$ be an arbitrary open covering of $X=X_{1} \times \ldots \times X_{k}$. From Corollaries (2.3) and (3.20) it follows that there exists a countable open family $\mathfrak{B}$ such that $\delta \mathfrak{B}<\delta \mathfrak{U}$ and $\cup \mathfrak{S} \supset X_{1}^{d} \times \ldots \times X_{k}^{d}$.

Let $H$ be an open set such that $X^{d} \times \ldots \times X_{k}^{d} \subset H \subset \bar{H} \subset \cup \mathfrak{B}$ and let $Z=X \backslash H$. From normality of $X$ it follows that there exist open subsets $P_{1}, \ldots, P_{k}$ of $Z$ such that $\bar{P}_{j} \subset G_{j}$, where $G_{j}=\left\{x \in Z: x_{j} \in X_{j} \backslash X_{j}^{d}\right\}$, and $\bigcup_{j=1}^{k} P_{j}=Z$. From the inductive assumption it follows that there exists a $\sigma$-discrete open covering $\mathfrak{U}_{j}$ of $\bar{P}_{j}$ such that $\delta \mathfrak{U}_{j}<\delta \mathfrak{U}$ for $j=1,2, \ldots, k$. The family $\mathfrak{H}=V \cup \bigcup_{j=1}^{k}\left\{U \cap P_{j} \cap(X \backslash \bar{H}): U \in \mathfrak{U}_{j}\right\}$ is an open, $\sigma$-discrete refinement of $\mathfrak{U}$. The proof of the part in brackets is similar.

E. Michael showed that if $2^{N_{0}}=\aleph_{1}$ then for $n=1,2, \ldots$, there exists a $\mathrm{GO}_{-}$-space $X(n)$ such that the Cartesian product $(X(n))^{n}$ is Lindelöf, while $(X(n))^{n+1}$ is paracompact but not Lindelöf (see [10], Example 1.4). Hence we cannot replace (b) of Corollary (3.22) by (b) of Corollary (3.20).

$\mathrm{S}$. Willard proved that if a space $X$ of the form $X=Y \times Z$ is paracompact, where $Y$ is separable and $Z$ has the Lindelöf property, then $X$ is a Lindelöf space (see [14]).

From this fact and Corollary (3.22) it follows that in the part of Corollaries (3.20) and (3.21) out of the brackets it is sufficient to assume that $X_{1}, X_{2}, \ldots, X_{n-1}$ are hereditarily Lindelöf spaces and $X_{n}$ is a Lindelöf space.

(3.23) Conollary. If $X_{i}$ is a $\mathrm{GO}_{\delta}$-space for $i=1,2, \ldots, n$ then the equivalence of (a) and (b) from Corollary (3.22) is independent of the usual axioms of set theory.

I am thankful to Professor R. Engelking for his help and suggestions in the preparation of this paper.

Added in proof. The notion of finite subparacompact spaces was introduced by T. A R countably subparacompact spaces, Pacific J. Math. (1973), pp. 209-213.

\section{References}

[1] K. Alstor and R. Engelking, Subparacompactiness and product spaces, Bull. Acad. Polon. Sci. Sór. Sci. Math. Astronom. Phys. 20 (1972), pp. 763-767.

R. Bing, Metrization of topological spaces, Canad. J. Math. 3 (1951), pp. 175-186.

[3] D. K. Burke, On subparacompact spaces, Proc. Amer. Math. Soc. 23 (1969), pp. $655-663$.

[4] R. Engelking, Outline of General Topology, Amsterdam 1968.

[5] R. W. Heath and E. Michael, A property of Sorgenfrey line, Composito Math. 23 (1971), pp. 185-188.

[6] K. Kuratowski, Topology, vol. I, New York-London-Warszawa 1966.
[7] D. J. Lutzer, On generalized ordered spaces, Dissert. Math. 89 (1971), pp. 1-36.

[8] - Another property of Sorgenfrey line, to appear. 
[9] E. Michael, The product of a normal space and a metrio space need not be normal, Bull. Amer. Math. Soc. 69 (1963), pp. 375-376.

[10] - Paracompactness and the Lindelof property in finite and countable Oartesian products, Composito Math. 23 (1971), pp. 199-214.

[11] T. Przymusiński, $A$ Lindelof space $X$ such that $X^{2}$ is normal but not paracompact, Fund. Math. 78 (1973), pp. 291-296.

[12] R. H. Sorgenfrey, On the topological product of paracompact spaces, Bull. Amer. Math. Soc. 53 (1947), pp. 631-632.

[13] L. A. Steen, A direct proof that the interval topology is collectionwise normal, Proc. Amer. Math. Soc. 24 (1970), pp. 727-728.

[14] S. Willard, Paracompactness in small products, Canad. Math. Bull. 14 (1971), p. 127.

INSTITUTE OF MATHEMATICS, POIISH ACADEMY OF SCIENCES

INSTYTUT MATEMATYCZNY POLSKIEJ AKADEMII NAUK

Accepté par la Rédaction le 10. \%. 1973

\section{An insertion theorem for real functions}

\section{by}

\author{
J. M. Boyte and E. P. Lane (Boone, North Carolina)
}

Abstract. Characterizations of countably paracompact spaces and of normal countably paracompact spaces in terms of insertion of extended real-valued semi-continuous and continuous functions are given.

Dowker [1] and Katĕtov [2] proved that a topological space $X$ is normal and countably paracompact if and only if for real-valued functions $f$ and $g$ defined on $X$ such that $f$ is lower semi-continuous and $g$ is upper semi-continuous and $g(x)<f(x)$ for each $x$ there exists a continuous realvalued function $h$ on $X$ such that $g(x)<h(x)<f(x)$ for each $x$. Mack [3] proved that a space is countably paracompact if and only if for each lower semi-continuous function $g$ on $X$ such that $g(x)>0$ for each $x$ there exist a lower semi-continuous function $l$ and an upper semi-continuous function $u$ such that $0<l(x) \leqslant u(x) \leqslant g(x)$ for each $x$. This note generalizes these results by using extended real-valued functions.

The abbreviations lsc (resp. usc) for lower semi-continuous (resp. upper semi-continuous) are used, and we write $g \leqslant f($ resp. $g<f$ ) in case $g(x) \leqslant f(x)$ (resp. $g(x)<f(x)$ ) for each $x$. Denote by $L$ (resp. $U$ ) the set of extended real-valued lsc (resp. usc) functions defined on $X$. If $f$ and $g$ of extended real-valued lsc (resp. usc) funs on $X$, we write $g \ll f$ in case $g \leqslant f$ and if either $g(x)$ or $f(x)$ is a real number, then $g(x)<f(x)$.

Trenomen. The following are equivalent:

(a) The space $X$ is normal and countably paracompact.

( $\beta)$ If $f \in L, g \in U$, and $g \ll f$, then there exist functions $f^{\prime} \in L$ and $g^{\prime} \in U$ such that $g \ll f^{\prime} \leqslant g^{\prime} \ll f$.

( $\gamma$ ) The space $X$ is normal, and if $f \in L, g \in U$ and $g \ll f$, then there exists a function $h \in L$ such that $g \ll h \ll f$.

( $\delta)$ If $f \in I, g \in U$, and $g \ll f$, then there exists an extended real-valued continuous function $b$ on $X$ such that $g \ll b \ll f$.

Proof. Observe that $(\delta)$ implies $(\beta)$ trivially. The proof that $(\beta)$ implies $(\alpha)$ is established as in the proof of Theorem 4 of [1]. In order to see that $(\gamma)$ implies $(\delta)$, let $f \in L, g \in U$, and $g \ll f$. By $(\gamma)$ there is a func- 